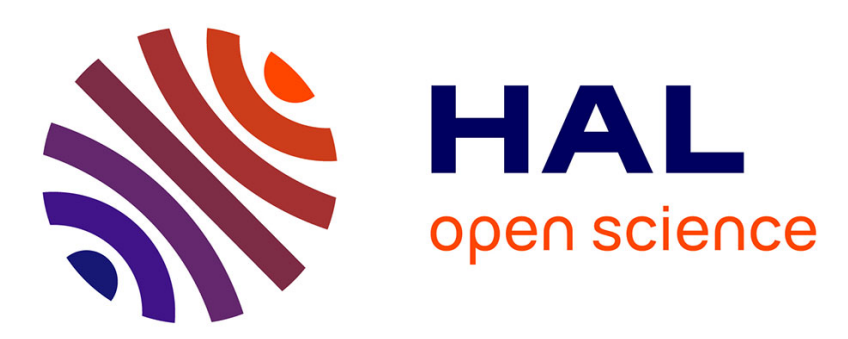

\title{
A quasi-optimal a priori error estimate for two-dimensional Signorini problem approximated by linear finite elements
}

Yves Renard

\section{- To cite this version:}

Yves Renard. A quasi-optimal a priori error estimate for two-dimensional Signorini problem approximated by linear finite elements. Comptes Rendus. Mathématique, 2012, 350, pp.325-328. 10.1016/j.crma.2012.01.024 . hal-00690522

\section{HAL Id: hal-00690522 \\ https://hal.science/hal-00690522}

Submitted on 19 Apr 2018

HAL is a multi-disciplinary open access archive for the deposit and dissemination of scientific research documents, whether they are published or not. The documents may come from teaching and research institutions in France or abroad, or from public or private research centers.
L'archive ouverte pluridisciplinaire HAL, est destinée au dépôt et à la diffusion de documents scientifiques de niveau recherche, publiés ou non, émanant des établissements d'enseignement et de recherche français ou étrangers, des laboratoires publics ou privés. 


\title{
A quasi-optimal a priori error estimate for the two-dimensional Signorini problem approximated by linear finite elements
}

\author{
Une estimation d'erreur quasi-optimale pour l'approximation par éléments finis du \\ problème de Signorini bidimensionnel
}

\author{
Yves Renard \\ Université de Lyon, CNRS, INSA-Lyon, ICJ UMR5208, 69621 Villeurbanne, France
}

\begin{abstract}
A B S T R A C T
The aim of this Note is to present a quasi-optimal a priori error estimate for the linear finite element approximation of the so-called two-dimensional Signorini problem, i.e. the equilibrium of a plane linearly elastic body in contact with a rigid foundation. Previous works on that subject give either non-optimal estimates or with a more restrictive supplementary condition on the solution.
\end{abstract}

\section{R É S U M É}

On présente dans cette Note une estimation optimale de l'erreur d'approximation par éléments finis affines du problème de Signorini, c'est à dire du problème de l'équilibre d'un corps élastique en contact avec une fondation rigide. Les travaux précédents sur ce sujet donnent soit des résultats non optimaux, soit avec des conditions supplémentaires plus contraignantes sur la solution.

\section{Introduction}

The a priori error estimate for the finite element approximation of Signorini's problem, i.e. the static contact problem of a linearly elastic structure (see [4]), has been addressed for the first time by J. Haslinger in [7]. Since then, it has been the subject of various works. If we limit ourselves to the approximation by linear finite elements, we can distinguish two types of approximations. The first one consists in directly approximating the variational inequality describing the Signorini problem, the only unknown being the elastic displacement [7,8,11]. The second approach, sometimes called hybrid formulation, involves a Lagrange multiplier to enforce the non-penetration in some weaker sense $[9,2,12,10]$.

A priori error estimate in the $H^{1}$-norm for the displacement of order $h^{3 / 4}$ have been established in [8], for the direct approximation, and in [2], for the hybrid one, under $H^{2}$ regularity assumption on the solution. The order $h$ has been shown in $[9,2,12]$ with an additional regularity assumption on the contact stress $\left(H^{1}\left(\Gamma_{C}\right)\right.$, where $\Gamma_{C}$ is the contact boundary). Note that an order $h$ was early proposed in [11] but with an erroneous estimate in negative norm for the Lagrange interpolation operator. More optimal estimates have been proven with the additional assumption that the number of transitions between contact and non-contact is finite on the contact boundary. Order $h \sqrt{|\log h|}$ in obtained in [1] and $h \sqrt[4]{|\log h|}$ in [2], and

E-mail address: Yves.Renard@insa-lyon.fr. 




Fig. 1. Linearly elastic body $\Omega$ in contact with a rigid foundation.

finally $h$ in [10] where the result is also extended to three-dimensional problems. This condition may seem reasonable at first glance. However, there exist some infinitely oscillating solutions to the elasticity problem in the simple situation of a transition between Neumann and Dirichlet conditions [6]. Thus, infinitely oscillatory situation cannot be a priori excluded.

The result presented here concerns the direct approximation and is quasi-optimal in the sense that the only requirement for the solution to obtain the $O(h)$ estimate is the $H^{2+\varepsilon}$ regularity for an arbitrary small $\varepsilon$. The argument is limited to two-dimensional domains.

\section{Signorini's problem}

Let $\Omega \subset \mathbb{R}^{2}$ be a bounded regular domain which represents the reference configuration of a linearly elastic body submitted to a Neumann condition on $\Gamma_{N}$, a Dirichlet condition on $\Gamma_{D}$ and a unilateral contact condition on $\Gamma_{C}$ between the body and a rigid foundation, where $\Gamma_{N}, \Gamma_{D}$ and $\Gamma_{C}$ are non-overlapping open parts of $\partial \Omega$, the boundary of $\Omega$ (see Fig. 1).

The displacement $u(t, x)$ of the body obeys the following equations:

$$
-\operatorname{div} \sigma(u)=f, \quad \text { in } \Omega, \quad \sigma(u)=\mathscr{A} \varepsilon(u), \quad \text { in } \Omega, \quad \sigma(u) \mathbf{n}=g, \quad \text { on } \Gamma_{N}, \quad u=0, \quad \text { on } \Gamma_{D},
$$

where $\sigma(u)$ is the stress tensor, $\varepsilon(u)=\left(\nabla u+\nabla u^{T}\right) / 2$ is the linearized strain tensor, $\mathscr{A}$ is the fourth order elasticity tensor which satisfies usual conditions of symmetry, coercivity and boundedness, $\mathbf{n}$ is the outward unit normal to $\Omega$ on $\partial \Omega$ and $f, g$ are given force densities. On $\Gamma_{C}$, it is usual to decompose the displacement and the stress in normal and tangential components as follows, assuming $\Gamma_{\mathrm{C}}$ to have the $\mathscr{C}^{1}$ regularity:

$$
u_{N}=u . \mathbf{n}, \quad u_{T}=u-u_{N} \mathbf{n}, \quad \sigma_{N}(u)=(\sigma(u) \mathbf{n}) . \mathbf{n}, \quad \sigma_{T}(u)=\sigma(u) \mathbf{n}-\sigma_{N}(u) \mathbf{n} .
$$

Assuming also that there is no initial gap between the solid and the rigid foundation, the frictionless unilateral contact condition is expressed by the following complementary condition:

$$
u_{N} \leqslant 0, \quad \sigma_{N}(u) \leqslant 0, \quad u_{N} \sigma_{N}(u)=0, \quad \sigma_{T}=0 .
$$

A classical weak form (see [4]) can be obtained introducing

$$
\begin{aligned}
& V=\left\{v \in H^{1}\left(\Omega ; \mathbb{R}^{d}\right), v=0 \text { on } \Gamma_{D}\right\}, \quad K=\left\{v \in V: v_{N} \leqslant 0 \text { on } \Gamma_{C}\right\}, \\
& a(u, v)=\int_{\Omega} \mathscr{A} \varepsilon(u): \varepsilon(v) \mathrm{d} x, \quad l(v)=\int_{\Omega} f . v \mathrm{~d} x+\int_{\Gamma_{N}} g . v \mathrm{~d} \Gamma .
\end{aligned}
$$

Problem (1)-(2) is then formally equivalent to the variational inequality

Find $u \in K$ satisfying

$$
a(u, v-u) \geqslant l(v-u), \quad \forall v \in K .
$$

\section{Finite element discretization}

A linear finite element approximation of Problem (3) is obtained given a regular family of triangulations $\mathscr{T}^{h}$ of $\Omega$ (in the sense of Ciarlet [3]). For the sake of simplicity, we assume $\Omega$ to be polygonal (which means that $\Gamma_{\mathrm{C}}$ is a line segment). We consider the classical finite element space

$$
V^{h}=\left\{v^{h} \in \mathscr{C}^{0}(\bar{\Omega}):\left.v\right|_{T} \in P^{1}(T) \forall T \in \mathscr{T}^{h}, v^{h}=0 \text { on } \Gamma_{D}\right\},
$$

and the discrete convex set $K^{h}=K \cap V^{h}$ of admissible displacements. Then, the finite element approximation of Problem (3) reads as

$$
\begin{aligned}
& \text { Find } u^{h} \in K^{h} \text { satisfying } \\
& a\left(u^{h}, v^{h}-u^{h}\right) \geqslant l\left(v^{h}-u^{h}\right), \quad \forall v^{h} \in K^{h} .
\end{aligned}
$$

We consider the following classical adaptation of Falk's lemma (see [7,8,11]): 
Lemma 3.1. Provided $f \in L^{2}(\Omega), g \in L^{2}\left(\Gamma_{N}\right), a(\cdot, \cdot)$ coercive, for $u$ solution to (3) with $\sigma_{N} \in L^{2}\left(\Gamma_{C}\right)$ and $u^{h}$ solution to (4), there exists $C>0$ a constant such that

$$
\left.\left\|u-u^{h \|}{ }_{1, \Omega} \leqslant C \inf _{v^{h} \in K^{h}}\right\| u-v^{h \|}{ }_{1, \Omega}+\int_{\Gamma_{C}} \sigma_{N} v_{N}^{h} \mathrm{~d} \Gamma\right)+C \inf _{v \in K} \int_{\Gamma_{C}} \sigma_{N}\left(v_{N}-u_{N}^{h}\right) \mathrm{d} \Gamma .
$$

Proof. We give the proof for the self-consistency of the Note. Denoting $\alpha>0$ the coercivity constant of $a(\cdot, \cdot)$ one has

$$
\alpha^{\|} u-u_{1, \Omega}^{h \|^{2}} \leqslant a\left(u-u^{h}, u-u^{h}\right)=a(u, u)+a\left(u^{h}, u^{h}\right)-a\left(u, u^{h}\right)-a\left(u^{h}, u\right) .
$$

Using the two variational inequalities, one deduces for arbitrary $v \in K$ and $v^{h} \in K^{h}$

$$
\alpha^{\|} u-u_{1, \Omega}^{h \|^{2}} \leqslant a\left(u, v-u^{h}\right)-l\left(v-u^{h}\right)+a\left(u, v^{h}-u\right)-l\left(v^{h}-u\right)+a\left(u^{h}-u, v^{h}-u\right) .
$$

Using now a Green formula (see [11]) one obtains $a(u, v)-l(v)={ }_{\Gamma_{C}} \sigma_{N} v_{N} \mathrm{~d} \Gamma$. From this and the complementarity condition, which gives $\sigma_{N} u_{N}=0$ a.e. on $\Gamma_{C}$, one obtains

$$
\alpha\left\|u-u^{h \|_{1, \Omega}^{2}} \leqslant \int_{\Gamma_{C}} \sigma_{N}\left(v_{N}-u_{N}^{h}\right) \mathrm{d} \Gamma+\int_{\Gamma_{C}} \sigma_{N} v_{N}^{h} \mathrm{~d} \Gamma+M^{\|} u-u^{h \|}{ }_{1, \Omega}\right\| u-v^{h \|}{ }_{1, \Omega},
$$

where $M>0$ is the continuity constant of $a(\cdot, \cdot)$. One can conclude with Young's inequality.

\section{Quasi-optimal error estimate}

Theorem 4.1. Provided $f \in L^{2}(\Omega), g \in L^{2}\left(\Gamma_{N}\right), a(\cdot, \cdot)$ coercive, for $u$ solution to (3) with $u \in H^{2+\varepsilon}(\Omega)$ for some $\varepsilon>0$ and $u^{h}$ solution to (4), there exists $C>0$ a constant independent of $h$ such that

$$
\left\|u-u^{h \|}{ }_{1, \Omega} \leqslant C h\right\| u \|_{2+\varepsilon, \Omega} .
$$

Proof. Since $K^{h} \subset K$ the second infimum of (5) vanishes. Considering $\Pi^{h}: H^{2}(\Omega) \rightarrow V^{h}$ the classical Lagrange interpolation operator and since $\Pi^{h} u \in K^{h}$ one deduces from (5)

$$
\left.\left\|u-u_{1, \Omega}^{h \|_{1}^{2}} \leqslant C \quad\right\| u-\Pi^{h} u \|_{1, \Omega}^{2}+\int_{\Gamma_{C}} \sigma_{N}\left(\Pi^{h} u\right)_{N} \mathrm{~d} \Gamma\right),
$$

where, here and hereafter, $C>0$ denotes a constant whose value may vary from an occurrence to another but which is independent of $h$ and of a possibly considered element. Since a classical interpolation error estimate [3] gives $\| u-$ $\Pi^{h} u \|_{1, \Omega} \leqslant C h|u|_{2, \Omega}$ we can focus on the last term of (7). Let $\mathscr{E}^{h h}$ be the set of edges on $\Gamma_{C}$ such that $u_{N}(c)<0$ for $c$ one of the vertices of the edge. Then

$$
\int_{\Gamma_{C}} \sigma_{N}\left(\Pi^{h} u\right)_{N} \mathrm{~d} \Gamma=\int_{e \in \mathscr{E}^{h}} \int_{e} \sigma_{N}\left(\Pi^{h} u\right)_{N} \mathrm{~d} \Gamma,
$$

because $\left(\Pi^{h} u\right)_{N}$ vanishes on each edge for which $u_{N}(a)=0$ on the two vertices. Now, for $e \in \mathscr{E}^{h}$ and due to the complementarity condition, one has

$$
\begin{aligned}
\int_{e} \sigma_{N}\left(\Pi^{h} u\right)_{N} \mathrm{~d} \Gamma & =\int_{e} \sigma_{N}\left(\left(\Pi^{h} u\right)_{N}-u_{N}\right) \mathrm{d} \Gamma \leqslant\left\|\sigma_{N}\right\|_{0, e}\left\|\left(\Pi^{h} u\right)_{N}-u_{N}\right\|_{0, e} \\
& \left.\leqslant C h\left\|\sigma_{N}\right\|_{0, e}^{2}+\frac{1}{h}\left\|\left(\Pi^{h} u\right)_{N}-u_{N}\right\|_{0, e}^{2}\right) .
\end{aligned}
$$

A standard estimate (see [8]) gives $\sum_{e \in \mathscr{E} h} \frac{1}{h}\left\|\left(\Pi^{h} u\right)_{N}-u_{N}\right\|_{0, e}^{2} \leqslant \frac{1}{h}\left\|\left(\Pi^{h} u\right)_{N}-u_{N}\right\|_{0, \Gamma_{C}}^{2} \leqslant C h^{2}\|u\|_{2, \Omega}^{2}$. We use a scaling argument to estimate the remaining term $\left\|\sigma_{N}\right\|_{0, e}^{2}$. Let $\hat{e}=(0,1)$ be a reference segment and let us denote by $\hat{\sigma}_{N}$ the normal stress obtained from $e$ on $\hat{e}$ by a linear transformation. Since $u_{N}<0$ on one of the vertices of $e$ and due to the complementarity condition, there exists a small interval $(0, \varepsilon)$ on which $\hat{\sigma}_{N}$ is a.e. equal to 0 , eventually adapting the orientation of the linear transformation to ensure the correspondence of 0 with the vertex on which $u_{N}<0$. In particular, this means $\hat{\sigma}_{N} \in H_{0}^{1 / 2+\varepsilon}(\hat{e})$, where $H_{0}^{1 / 2+\varepsilon}(\hat{e})=\left\{v \in H^{1 / 2+\varepsilon}(\hat{e}), v(0)=0\right\}$. We will prove later on that the semi-norm 


$$
\left.|v|_{1 / 2+\varepsilon, \hat{e}}=\int_{\hat{e}} \int_{\hat{e}} \frac{(v(\hat{x})-v(\hat{y}))^{2}}{|\hat{x}-\hat{y}|^{2+2 \varepsilon}} \mathrm{d} \hat{x} \mathrm{~d} \hat{y}\right)^{1 / 2}
$$

is equivalent on $H_{0}^{1 / 2+\varepsilon}(\hat{e})$ to the classical norm $\|v\|_{1 / 2+\varepsilon, \hat{e}}=\left(\|v\|_{0, \hat{e}}^{2}+|v|_{1 / 2+\varepsilon, \hat{e}}^{2}\right)^{1 / 2}$. This leads to

$$
\left\|\sigma_{N}\right\|_{0, e}^{2}=|e|\left\|\hat{\sigma}_{N}\right\|_{0, \hat{e}}^{2} \leqslant C h\left|\hat{\sigma}_{N}\right|_{1 / 2+\varepsilon, \hat{e}}^{2}=C h^{1+2 \varepsilon}\left|\sigma_{N}\right|_{1 / 2, e}^{2} .
$$

Since $\sum_{e \in \mathscr{E}^{h}}\left|\sigma_{N}\right|_{1 / 2+\varepsilon, e}^{2} \leqslant\left|\sigma_{N}\right|_{1 / 2+\varepsilon, \Gamma_{C}}^{2} \leqslant C\|u\|_{2+\varepsilon, \Omega}^{2}$, one finally obtains (6). The proof of equivalence on $H_{0}^{1 / 2+\varepsilon}(\hat{e})$ between the two norms $|v|_{1 / 2+\varepsilon, \hat{e}}$ and $\|v\|_{1 / 2+\varepsilon, \hat{e}}$ is a straightforward application of the Petree-Tartar lemma we recall here (see [5] for instance).

Lemma 4.2. Let $X, Y, Z$ be three Banach spaces, $A \in \mathscr{L}(X, Y)$ be injective and $T \in \mathscr{L}(X, Z)$ be compact, if there exists $c>0$ a constant such that $c\|x\|_{X} \leqslant\|A x\|_{Y}+\|T x\|_{Z} \quad \forall x \in X$ then the range of $A$ is closed or, equivalently, there exists $\alpha>0$ a constant such that

$$
\alpha\|x\|_{X} \leqslant\|A x\|_{Y} \quad \forall x \in X .
$$

The equivalence is obtained by taking $X=H_{0}^{1 / 2+\varepsilon}(\hat{e}), Y=L^{2}(\hat{e} \times \hat{e}), Z=L^{2}(\hat{e}), A v=\frac{v(\hat{x})-v(\hat{y})}{|\hat{x}-\hat{y}|^{1+\varepsilon}}$ and $T$ the compact injection between $X$ and $Z$. $A$ is injective since $A v=0$ implies $v$ is a constant a.e. on $\hat{e}$ and since the only constant in $H_{0}^{1 / 2+\varepsilon}(\hat{e})$ is zero.

\section{References}

[1] F. Ben Belgacem, Numerical simulation of some variational inequalities arisen from unilateral contact problems by the finite element method, SIAM J. Numer. Anal. 37 (4) (2000) 1198-1216.

[2] F. Ben Belgacem, Y. Renard, Hybrid finite element methods for Signorini's problem, Math. Comp. 72 (2003) $1117-1145$.

[3] P.G. Ciarlet, The Finite Element Method for Elliptic Problems, Studies in Mathematics and Its Applications, North-Holland Publishing, 1978.

[4] G. Duvaut, J.L. Lions, Les inéquations en mécanique et en physique, Dunod, Paris, 1972.

[5] A. Ern, J.-L. Guermond, Éléments finis: théorie, applications, mise en œuvre, Mathématiques et Applications, vol. 36, SMAI, Springer-Verlag, 2002.

[6] P. Grisvard, Singularities in Boundary Value Problems, Masson, 1992.

[7] J. Haslinger, Finite element analysis for unilateral problems with obstacles on the boundary, Appl. Math. 22 (3) (1977) $180-188$.

[8] J. Haslinger, I. Hlaváček, J. Nečas, Numerical Methods for Unilateral Problems in Solids Mechanics, in: Handbook of Numerical Analysis, vol. IV, Elsevier Science, 1996, pp. 313-485.

[9] P. Hild, Numerical implementation of two nonconforming finite element methods for unilateral contact, Comput. Meth. Appl. Mech. Eng. 184 (1) (2000) 99-123.

[10] S. Hüeber, B.I. Wohlmuth, An optimal a priori error estimate for nonlinear multibody contact problems, SIAM J. Numer. Anal. 43 (1) (2005) 156-173.

[11] N. Kikuchi, J.T. Oden, Contact Problems in Elasticity, SIAM, 1988.

[12] P. Laborde, Y. Renard, Fixed point strategies for elastostatic frictional contact problems, Math. Methods Appl. Sci. 31 (2008) $415-441$. 